\title{
Correction to: Salidroside exhibits anti-dengue virus activity by upregulating host innate immune factors
}

\author{
Navita Sharma ${ }^{1}$ K. P. Mishra ${ }^{1}$ Lilly Ganju ${ }^{1}$
}

Published online: 9 December 2021

(c) Springer-Verlag GmbH Austria, part of Springer Nature 2021

\section{Correction to: Arch Virol (2016) 161:3331-3344 https://doi.org/10.1007/s00705-016-3034-1}

The authors would like to make the readers aware of an error in the paper entitled "Salidroside exhibit anti-dengue virus activity by upregulating host innate immune factors" by Sharma et al. (Archives of Virology 2016 Dec;161(12):33313344; https://doi.org/10.1007/s00705-016-3034-1.

It was brought to our notice that Western blot band of beta actin of IRF-3 in Figure 5E is identical with the beta actin band of Fig 3A. The authors confirm that, in Fig. 5E, the beta actin blot was incorrectly pasted while compiling the figure for representation of IRF-3. We regret this inadvertent mistake and replacing the beta actin blot of figure 5E. The original blot of beta actin and revised Fig 5E are attached herewith to be published as erratum. We confirm that the outcome of the study was in no way affected by this error and does not impact either the results or discussion. The corrected version of the beta actin blot of Fig 5E for IRF-3 is provided here.

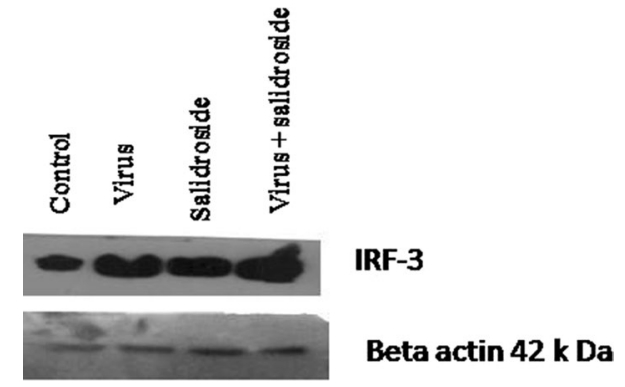

Fig. 5 Effect of salidroside on host innate immune factors. e. Immunoblot of IRF-3 expression in DENV infected THP-1 cells after salidroside treatment. The revised beta actin band are provided.

Publisher's Note Springer Nature remains neutral with regard to jurisdictional claims in published maps and institutional affiliations.
The original article can be found online at https://doi.org/10.1007/ s00705-016-3034-1.

Navita Sharma

kpmpgi@rediffmail.com

1 Immunomodulation Laboratory, Defence Institute of Physiology and Allied Sciences (DIPAS), DRDO, Lucknow Road, Timarpur, Delhi 110054, India 